\title{
Mechanisms of fatigue-crack initiation and their impact on fatigue life of AISi7 die-cast components
}

\author{
Sabine Redik ${ }^{\mathrm{a}}$, Markus Tauscher and Florian Grün \\ Chair of Mechanical Engineering, Montanuniversität Leoben, Austria
}

\begin{abstract}
In the course of the present study, in-situ observations of crack initiation and crack growth of naturally induced cracks in cyclically loaded specimens along with conventional fatigue tests and fracture surface analyses were performed. The specimens used were taken from different sampling positions of standard and HIPed aluminum-diecast engine blocks, with different cooling conditions. In one sampling position within the standard engine block microporosity was able to form, acting as a source for fatigue-crack initiation. While in the absence of microporosity, as observed in specimens taken from HIPed components, crack initiation occured via slip band mechanism. If material defects such as pores were present, premature crack initiation reduced the fatigue life yielding a lower fatigue life and fatigue strength than specimens where cracks formed by slip band mechanism. For cracks formed at pores, the pore size is the determining factor for fatigue behavior. While for cracks initiated via slip band mechanism fatigue strength is a function of the local material strength.
\end{abstract}

\section{Introduction}

Fatigue life of aluminum-die-cast components is determined by their microstructure, predominantly through its influence on fatigue-crack initiation. The casting process of aluminum-die-cast components naturally implies local differences in microstructure and therefore causes different material behavior.

\section{Experimental procedure}

In the present study in-situ observations of crack initiation and growth of naturally induced cracks from pores and via slip band mechanism were performed under rotating-bending conditions in order to evaluate the different fatigue-crack initiation mechanisms. In addition, conventional fatigue tests using specimens from standard and HIPed AlSi7MgCu0.5-T6 die-cast engine blocks with subsequent fracture surface analyses were performed to quantify the effect of the fatigue-crack initiation mechanism. To derive a model for fatigue strength based on microstructure with broad applicability, additional data taken from literature were used.

\footnotetext{
${ }^{\text {a }}$ Corresponding author: sabine.redik@unileoben.ac . at
}

This is an Open Access article distributed under the terms of the Creative Commons Attribution License 4.0, which permits unrestricted use, distribution, and reproduction in any medium, provided the original work is properly cited. 

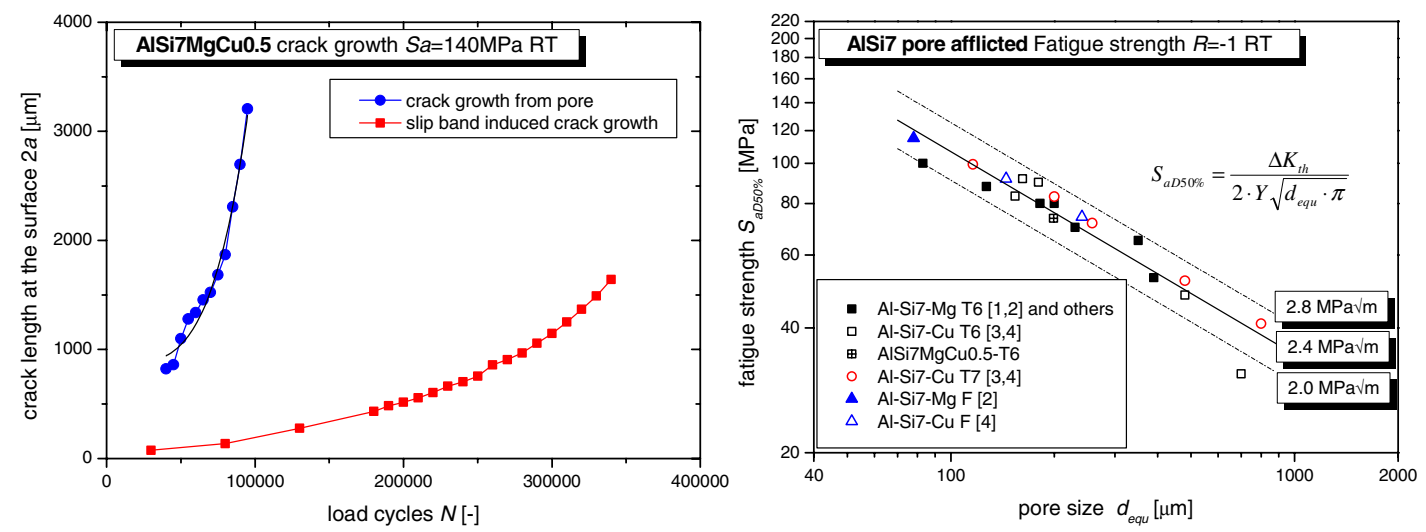

Figure 1. In-situ observation of crack growth of naturally induced cracks (left), fracture mechanical model for fatigue strength prediction based on pore size.

\section{Results}

The in-situ observations revealed that slip band induced and defect induced fracture origin from pores differ in their initial crack length. Figure 1 (left) shows crack growth of naturally induced cracks from a pore and via slip band mechanism. The crack induced via slip band mechanism starts at zero crack length while the crack originated from a pore exhibits an initial crack length in the size of the crack initiating pore. Therefore the fatigue strength for defect induced fracture can be modelled using a fracture mechanical approach. In Fig. 1 (right) the fatigue strength $S_{a D 50 \%}$, is plotted against the mean pore size $d_{\text {equ }}$. The data, taken from literature [1-4] as well as from own fatigue tests, include AlSi7alloys with varying $\mathrm{Mg}$ - and $\mathrm{Cu}$-content as well as different heat treatment conditions (T6, T7, F). All data lie within the same scatter band and can be described using a Kitagawa-model. The corresponding threshold value $\Delta K_{t h}$ is in the range of 2.0 to $2.8 \mathrm{MPa} \sqrt{ } \mathrm{m}$.

For slip band induced fracture origin it was found that fatigue strength can be estimated based on the materials yield strength, since the yield strength correlates with the resistance toward dislocation movement necessary for the formation of fatigue slip bands. This result was based on data from literature $[1,3,5]$ of different Sr-refined AlSi7-alloys with varying $\mathrm{Mg}$ - and $\mathrm{Cu}$-content in $\mathrm{T} 6$ and $\mathrm{T} 7$ heat treatment conditions in addition to own fatigue results using specimens from HIPed components.

\section{Conclusion}

Pores act as cracks with an initial crack size in the magnitude of the pore size. For pore afflicted AlSi7alloys fatigue strength is only dependant on pore size and can be described using a fracture mechanical approach. Alloy composition in terms of $\mathrm{Mg}$ - and $\mathrm{Cu}$-content as well as heat treatment conditions are of negligible influence. While for pore-free Sr-refined AlSi7-alloys with heat treatment conditions including the process of solution annealing, the fatigue strength is a function of the materials yield strength.

\section{References}

[1] J.Z. Yi, Y.X. Gao, P.D. Lee, T.C. Lindley, Metal. and Mat. Trans. 37B, p.301-311 (2006)

[2] M.J. Couper, A.E. Neeson, J.R. Griffiths, Fatig. Fract. Eng. Mater. Struct. 13, p.213-227 (1990) 
[3] J.M. Boileau, J.E. Allison, Metal. and Mat. Trans. 34A, p.1807-1820 (2003)

[4] R. Gonzales, A. Gonzales, J. Talamantes-Silva, S. Valtierra, R.D. Mercado-Solis, N.F. GarzaMontes-de-Oca, R. Colas, Int. J. Fatigue 54, p.118-126 (2013)

[5] M. Brochu, Y. Verreman, F. Ajersch, D. Bouchard, Int. J. Fatigue 32, p.1233-1242 (2010) 